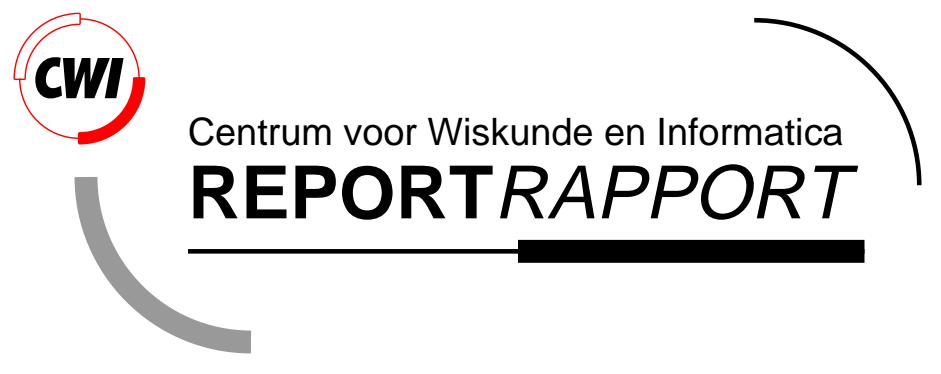

Asymptotic behavior of Generalized Processor Sharing with long-tailed traffic sources

S.C. Borst, O.J. Boxma, P.R. Jelenkovic

Probability, Networks and Algorithms (PNA)

PNA-R9916 December 31, 1999 
Report PNA-R9916

ISSN 1386-3711

CWI

P.O. Box 94079

1090 GB Amsterdam

The Netherlands

$\mathrm{CWI}$ is the National Research Institute for Mathematics and Computer Science. CWI is part of the Stichting Mathematisch Centrum (SMC), the Dutch foundation for promotion of mathematics and computer science and their applications.

SMC is sponsored by the Netherlands Organization for Scientific Research (NWO). CWI is a member of ERCIM, the European Research Consortium for Informatics and Mathematics.

Copyright @ Stichting Mathematisch Centrum P.O. Box 94079, 1090 GB Amsterdam (NL) Kruislaan 413, 1098 SJ Amsterdam (NL) Telephone +3120 5929333 Telefax +3120 5924199 


\title{
Asymptotic Behavior of Generalized Processor Sharing with Long-Tailed Traffic Sources
}

\author{
Sem Borst ${ }^{*, *, \dagger}$, Onno Boxma ${ }^{*, *}$, Predrag Jelenković ${ }^{\ddagger}$ \\ ${ }^{*} C W I$ \\ P.O. Box 94079, 1090 GB Amsterdam, The Netherlands \\ ** Department of Mathematics $\& 3$ Computing Science \\ Eindhoven University of Technology \\ P.O. Box 513, 5600 MB Eindhoven, The Netherlands \\ ${ }^{\dagger}$ Bell Laboratories, Lucent Technologies \\ P.O. Box 636, Murray Hill, NJ 07974, USA \\ ${ }^{\ddagger}$ Department of Electrical Engineering \\ Columbia University \\ New York, NY 10027, USA
}

\begin{abstract}
We analyze the asymptotic behavior of long-tailed traffic sources under the Generalized Processor Sharing (GPS) discipline. GPS-based scheduling algorithms, such as Weighted Fair Queueing, have emerged as an important mechanism for achieving differentiated quality-ofservice in integrated-services networks.

Under certain conditions, we prove that in an asymptotic sense an individual source with longtailed traffic characteristics is effectively served at a constant rate, which may be interpreted as the maximum feasible average rate for that source to be stable. Thus, asymptotically, the source is only affected by the traffic characteristics of the other sources through their average rate. In particular, the source is essentially immune from excessive activity of sources with 'heavier'-tailed traffic characteristics. This suggests that GPS-based scheduling algorithms provide an effective mechanism for extracting high multiplexing gains, while protecting individual connections.
\end{abstract}

1991 Mathematics Subject Classification: 60K25 (primary), 68M20, 90B12, 90B22 (secondary). Keywords and Phrases: Generalized Processor Sharing (GPS), long-tailed, queue length asymptotics, regular variation, subexponential, Weighted Fair Queueing (WFQ).

Note: Work of the first two authors carried out in part under the project PNA2.1 "Communication and Computer Networks". 


\section{Introduction}

Statistical data analysis has provided convincing evidence of long-tailed (subexponential [20]) traffic characteristics in high-speed communication networks. Early indications of the longrange dependence of Ethernet traffic, attributed to long-tailed file size distributions, were reported in [29]. Long-tailed characteristics of the scene length distribution of MPEG video streams were explored in $[21,26]$.

These empirical findings have encouraged theoretical developments in the modeling and queueing analysis of long-tailed traffic phenomena. Despite significant progress, however, the practical implications are not yet thoroughly understood, in particular issues relating to control and priority mechanisms in the network. To gain a better understanding of those issues, the present paper analyzes the queueing behavior of long-tailed traffic sources under the Generalized Processor Sharing (GPS) discipline. As a design paradigm, GPS is at the heart of commonly-used scheduling algorithms for high-speed switches, such as Weighted Fair Queueing, see for instance Parekh \& Gallager [32, 33].

A basic approach in the analysis of long-tailed traffic phenomena is the use of fluid models with long-tailed arrival processes (e.g. On/Off sources with long-tailed On-periods). Fluid models are closely related to the ordinary single-server queue, thus bringing within reach the classical results on regularly-varying (Cohen [18]) or subexponential (Pakes [31], Veraverbeke [35]) behavior of the service and waiting-time distribution in the GI/G/1 queue. Those results are immediately applicable in the case of a single long-tailed arrival stream, see Boxma [10] and Choudhury \& Whitt [16]. They are also of use when a single long-tailed stream is multiplexed with exponential streams, see Boxma [11], Jelenković \& Lazar [24], and Rolski et al. [34].

The queueing analysis of fluid models with multiple long-tailed arrival streams is fundamentally more difficult due to the complex dependency structure in the aggregate arrival process, see for instance Heath et al. [22]. Recently, Agrawal et al. [2] obtained interesting partial results. General bounds were derived in Choudhury \& Whitt [16]. Boxma [11] and Jelenković \& Lazar [24] studied the limiting process obtained by multiplexing an infinite number of On-Off sources with regularly-varying and subexponential On-periods, respectively. We refer to Boxma \& Dumas [14] for a comprehensive survey on fluid queues with long-tailed arrival processes. See also Jelenković [23] for an extensive list of references on subexponential queueing models. As mentioned above, the impact of priority mechanisms on long-tailed traffic phenomena has received relatively little attention. Some recent studies have investigated the effect of the scheduling discipline on the waiting-time distribution in the classical M/G/1 queue, see for instance Anantharam [3]. For FCFS, it is well-known [18] that the waiting-time tail is regularly varying of index $1-\nu$ iff the service time tail is regularly varying of index $-\nu$. For LCFS preemptive resume as well as for Processor Sharing, the waiting-time tail turns out to be regularly varying of the same index as the service time tail [12], [39], although with different 
pre-factors. In the case of Processor Sharing with several customer classes, Zwart [38] recently showed that the sojourn time distribution of a class- $i$ customer is regularly varying of index $-\nu_{i}$ iff the service time distribution of that class is regularly varying of index $-\nu_{i}$, regardless of the service time distributions of the other classes. In contrast, for two customer classes with ordinary non-preemptive priority, the tail behavior of the waiting- and sojourn time distributions is determined by the heaviest of the (regularly-varying) service time distributions $[1,13]$.

In the present paper, we consider the Generalized Processor Sharing (GPS) discipline. GPSbased scheduling algorithms, such as Weighted Fair Queueing, have emerged as an important mechanism for achieving differentiated quality-of-service in integrated-services networks. The queueing analysis of GPS is extremely difficult. Interesting partial results for exponential traffic models were obtained in Bertsimas et al. [5], Dupuis \& Ramanan [19], Massoulié [30], Zhang [36], and Zhang et al. [37].

Here, we focus on non-exponential traffic models. Extending the results from [7], we show that, under certain conditions, an individual source with long-tailed traffic characteristics is effectively served at a constant rate, which may be interpreted as the critical mean rate for stability. This is strongly reminiscent of the reduced-load equivalence established by Agrawal et al. [2]. In particular, the source is largely insensitive to excessive activity of sources with 'heavier'-tailed traffic characteristics. This insensitivity property starkly contrasts with a recent result in [9], which shows that in other scenarios a source may be strongly affected by the activity of 'heavier'-tailed sources, and may inherit their traffic characteristics, causing induced burstiness. The sharp dichotomy in qualitative behavior illustrates the crucial importance of the weight parameters in protecting individual connections while extracting multiplexing gains. The remainder of the paper is organized as follows. In Section 2, we present a detailed model description. In Section 3, we briefly discuss some stability issues, and introduce a stabilityrelated notion which will play a crucial role in the analysis. General lower and upper bounds on the buffer content of an individual source are derived in Section 4. We then show, in Section 5, that for long-tailed traffic characteristics, the lower and upper bounds have the same asymptotic behavior, yielding exact asymptotic results. In Section 6, we make some concluding remarks.

\section{Model description}

Consider $N$ sources sharing a link of unit rate. Traffic from the sources is served in accordance with the Generalized Processor Sharing (GPS) discipline, which operates as follows. There are weights $\phi_{1}, \ldots, \phi_{N}$ associated with each of the sources, with $\sum_{i=1}^{N} \phi_{i}=1$. Denote by $V_{i}(t)$ the buffer content of source $i$ at time $t$. Let $\mathbf{V}_{i}$ be a stochastic variable with as distribution the limiting distribution of $V_{i}(t)$ for $t \rightarrow \infty$ (assuming it exists). If all the sources are backlogged at 
time $t$, i.e., $V_{i}(t)>0$ for all $i=1, \ldots, N$, then source $i$ is served at rate $\phi_{i}$. If some of the sources are not backlogged, however, then the excess capacity is redistributed among the backlogged sources in proportion to their respective weights. We refer to Dupuis \& Ramanan [19] for a formal description of the evolution of the buffer content process.

Denote by $A_{i}(r, t)$ the amount of work generated by source $i$ during the time interval $(r, t]$, and assume that $A_{i}$ is a stationary process. Define $B_{i}(r, t)$ as the amount of service received by source $i$ during $(r, t]$. Then the following identity relation holds

$$
V_{i}(t)=V_{i}(r)+A_{i}(r, t)-B_{i}(r, t)
$$

for all $0 \leq r \leq t$

Remark 2.1 Although we use the term 'buffer content' to indicate the workload, we do not make any particular assumptions where traffic physically resides while waiting to be served. Using flow control algorithms, backlogged sources may for example be instructed to feed traffic into the network only at a rate comparable to the actual service rate so as to avoid excessive buffer overflow due to congestion.

Before describing the traffic model, we first introduce some further notation. For any two real functions $g(\cdot)$ and $h(\cdot)$, we use the notational convention $g(x) \sim h(x)$ as $x \rightarrow \infty$ to denote $\lim _{x \rightarrow \infty} g(x) / h(x)=1$, or equivalently, $g(x)=h(x)(1+o(1))$ as $x \rightarrow \infty$. For any stochastic variable $\mathbf{X}$ with distribution function $F(\cdot), \mathbb{E} \mathbf{X}<\infty$, denote by $F^{r}(\cdot)$ the distribution function of the residual lifetime of $\mathbf{X}$, i.e., $F^{r}(x)=\frac{1}{\mathbb{E} \mathbf{X}} \int_{0}^{x}(1-F(y)) \mathrm{d} y$.

The classes of long-tailed, subexponential, intermediately regularly varying, and dominatedly varying distributions are denoted with the symbols $\mathcal{L}, \mathcal{S}, \mathcal{I} \mathcal{R}$, and $\mathcal{D} \mathcal{R}$, respectively. The definitions of these classes are given in Appendix A.

For any $c \geq 0$, denote by $V_{i}^{c}(t):=\sup _{0 \leq r \leq t}\left\{A_{i}(r, t)-c(t-r)\right\}$ the buffer content of source $i$ at time $t$ if it were served in isolation at rate $c$ (assuming $V_{i}^{c}(0)=0$ ). Denote by $\rho_{i}$ the traffic intensity of source $i$. For $c>\rho_{i}$, let $\mathbf{V}_{i}^{c}$ be a stochastic variable with as distribution the limiting distribution of $V_{i}^{c}(t)$ for $t \rightarrow \infty$. Define $W_{i}^{c}(x):=\mathbb{P}\left\{\mathbf{V}_{i}^{c} \leq x\right\}$ and $\bar{W}_{i}^{c}(x):=1-W_{i}^{c}(x)$. In the next sections, we will analyze the asymptotic behavior of sources $i$ where the function $\bar{W}_{i}^{c}(\cdot)$ satisfies the following three properties for some value of $\sigma_{i}>\rho_{i}$.

Property 2.1 For any $c \in\left(\rho_{i}, \sigma_{i}\right), W_{i}^{c}(\cdot) \in \mathcal{L}$, i.e.,

$$
\lim _{x \rightarrow \infty} \frac{\bar{W}_{i}^{c}(x-y)}{\bar{W}_{i}^{c}(x)}=1, \text { for all real } y \text {. }
$$


Property 2.2 For any $c \in\left(\rho_{i}, \sigma_{i}\right), W_{i}^{c}(\cdot) \in \mathcal{D} \mathcal{R}$, i.e.,

$$
\limsup _{x \rightarrow \infty} \frac{\bar{W}_{i}^{c}(\eta x)}{\bar{W}_{i}^{c}(x)}=G_{i}^{c}(\eta)<\infty, \text { for some real } \eta \in(0,1)
$$

(which implies the property holds for all $\eta>0$ ).

Property 2.3 For any $c \in\left(\rho_{i}, \sigma_{i}\right)$,

$$
\limsup _{x \rightarrow \infty} \frac{\bar{W}_{i}^{c-\theta}(x)}{\bar{W}_{i}^{c}(x)}=H_{i}^{c}(\theta)<\infty, \text { for some real } \theta \in\left(0, c-\rho_{i}\right)
$$

(which implies the property holds for all $\theta>0$ small enough where we assume $\lim _{\theta \rightarrow 0} H_{i}^{c}(\theta)=1$ ).

We now describe two traffic scenarios where the function $\bar{W}_{i}^{c}(\cdot)$ satisfies the above three properties.

\subsection{Instantaneous arrivals}

Here, a source generates instantaneous traffic bursts according to independent renewal processes. The interarrival times between bursts of source $i$ are generally distributed with mean $1 / \lambda_{i}$. The burst sizes of source $i$ have distribution $B_{i}(\cdot)$ with mean $\beta_{i}$. Thus, the traffic intensity of source $i$ is $\rho_{i}=\lambda_{i} \beta_{i}$. Let $\mathbf{B}_{i}^{r}$ be a stochastic variable with distribution $B_{i}^{r}(\cdot)$.

The next result is immediate from Pakes [31].

Theorem 2.1 If $B_{i}^{r}(\cdot) \in \mathcal{S}$, and $\rho_{i}<c$, then

$$
\bar{W}_{i}^{c}(x) \sim \frac{\rho_{i}}{c-\rho_{i}} \mathbb{P}\left\{\mathbf{B}_{i}^{r}>x\right\} \quad \text { as } \quad x \rightarrow \infty .
$$

Thus, for any finite $\sigma_{i}>\rho_{i}, \bar{W}_{i}^{c}(\cdot)$ satisfies Properties 2.1 and 2.3 if $B_{i}^{r}(\cdot) \in \mathcal{S}$, and Property 2.2 if $B_{i}^{r}(\cdot) \in \mathcal{D} \mathcal{R} \cap \mathcal{L}$.

\section{$2.2 \quad$ On-Off processes}

Here, a source generates traffic according to independent On-Off processes, alternating between On- and Off-periods. The Off-periods of source $i$ are generally distributed with mean $1 / \lambda_{i}$. The On-periods of source $i$ have distribution $A_{i}(\cdot)$ with mean $\alpha_{i}$. While On, source $i$ produces traffic at a constant rate $r_{i}$, so the mean burst size is $\alpha_{i} r_{i}$. The fraction of time that source $i$ is Off is

$$
p_{i}=\frac{1 / \lambda_{i}}{1 / \lambda_{i}+\alpha_{i}}=\frac{1}{1+\lambda_{i} \alpha_{i}} .
$$

The traffic intensity of source $i$ is

$$
\rho_{i}=\left(1-p_{i}\right) r_{i}=\frac{\lambda_{i} \alpha_{i} r_{i}}{1+\lambda_{i} \alpha_{i}} .
$$


Let $\mathbf{A}_{i}^{r}$ be a stochastic variable with distribution $A_{i}^{r}(\cdot)$.

The next result is established in Jelenković \& Lazar [24].

Theorem 2.2 If $A_{i}^{r}(\cdot) \in \mathcal{S}$, and $\rho_{i}<c<r_{i}$, then

$$
\bar{W}_{i}^{c}(x) \sim p_{i} \frac{\rho_{i}}{c-\rho_{i}} \mathbb{P}\left\{\mathbf{A}_{i}^{r}>x /\left(r_{i}-c\right)\right\} \quad \text { as } \quad x \rightarrow \infty .
$$

Thus, for any $r_{i}>\sigma_{i}>\rho_{i}, \bar{W}_{i}^{c}(\cdot)$ satisfies Property 2.1 if $A_{i}^{r}(\cdot) \in \mathcal{S}$, Property 2.2 if $A_{i}^{r}(\cdot) \in$ $\mathcal{D} \mathcal{R} \cap \mathcal{L}$, and Property 2.3 if $A_{i}^{r}(\cdot) \in \mathcal{I} \mathcal{R}$.

\section{$3 \quad$ Stability issues}

We first briefly discuss some stability issues. If $\sum_{i=1}^{N} \rho_{i}<1$, then all the sources will be stable, since the GPS discipline is work-conserving. If $\sum_{i=1}^{N} \rho_{i}>1$, then at least one of the sources will be unstable, but others may still be stable. We now identify which sources are stable and which ones are unstable. To avoid technical subtleties, source $i$ is considered 'stable' if the mean service rate is $\rho_{i}$, see also Remark 3.1 below. For the ease of presentation, we assume the sources are indexed such that

$$
\frac{\rho_{1}}{\phi_{1}} \leq \ldots \leq \frac{\rho_{N}}{\phi_{N}}
$$

Proposition 3.1 With the above ordering, the set of stable sources is $S=\left\{1, \ldots, K^{*}\right\}$, with

$$
K^{*}=\max _{k=1, \ldots, N}\left\{k: \frac{\rho_{k}}{\phi_{k}} \leq \frac{1-\sum_{j=1}^{k-1} \rho_{j}}{\sum_{j=k}^{N} \phi_{j}}\right\} .
$$

\section{Proof}

See Appendix B.

It may be verified that $K^{*}=N$ (i.e. all the sources receive a stable service rate) iff $\sum_{i=1}^{N} \rho_{i} \leq 1$. By definition, each of the stable sources $i \in S$ receives a mean service rate $\rho_{i}$. Each of the unstable sources $i \notin S$ receives a mean service rate $\phi_{i} R<\rho_{i}$, with

$$
R=\frac{1}{\sum_{j \notin S} \phi_{j}}\left(1-\sum_{j \in S} \rho_{j}\right) \text {. }
$$


To understand the above formula, notice that the stable sources consume an average aggregate rate $\sum_{j \in S} \rho_{j}$, leaving an average rate $1-\sum_{j \in S} \rho_{j}$ for the unstable sources, which is shared in proportion to the weights $\phi_{i}$.

We now introduce a stability-related notion which will play a crucial role in the analysis. Define $\gamma_{i E}$ as the mean rate at which source $i$ would receive service if the sources $j \in E$ were to continuously claim their full share of the link rate (while the remaining sources $j \notin E$ still acted 'normally'). (With minor abuse of notation we write $\gamma_{i j}$ for $\gamma_{i\{j\}}$.) Now observe that the sources $j \in E$ would in fact show such greedy behavior if they were unstable (which they need not be in reality). So we may determine $\gamma_{i E}$ by forcing the sources $j \in E$ into the set of unstable sources, and then apply Proposition 3.1. The set of sources which would receive a stable service rate if the sources $j \in E$ were to continuously claim their full share of the link rate, is then $S_{E}=\left\{1, \ldots, K_{E}^{*}\right\} \backslash E$, with

$$
K_{E}^{*}=\max _{k=1, \ldots, N}\left\{k: \frac{\rho_{k}}{\phi_{k}} \leq \frac{1-\sum_{j=1}^{k-1} \rho_{j} \mathrm{I}_{\{j \notin E\}}}{\sum_{j=k}^{N} \phi_{j} \mathrm{I}_{\{j \notin E\}}+\sum_{j \in E} \phi_{j}}\right\} .
$$

Thus, $\gamma_{i E}=\rho_{i}$ for all $i \in S_{E}$, and $\gamma_{i E}=\phi_{i} R_{E}<\rho_{i}$ for all $i \notin S_{E}$, with

$$
R_{E}=\frac{1}{\sum_{j \notin S_{E}} \phi_{j}}\left(1-\sum_{j \in S_{E}} \rho_{j}\right) \text {. }
$$

To explain the above formula, observe that the sources $j \in S_{E}$ by definition receive an average aggregate rate $\sum_{j \in S_{E}} \rho_{j}$, leaving an average rate $1-\sum_{j \in S_{E}} \rho_{j}$ for the sources $j \notin S_{E}$, which is shared in proportion to the weights $\phi_{i}$.

Remark 3.1 For later purposes, we find it convenient to label source $i$ as 'stable' if the mean service rate is $\rho_{i}$. In fact, the latter condition is necessary for stability in the usual sense, but not entirely sufficient. A sufficient condition is $\rho_{i}<\gamma_{i i}$. Indeed, if the buffer of source $i$ never emptied, then it would receive a mean service rate $\gamma_{i i}$, so that $\gamma_{i i}$ is the critical mean rate for stability.

\section{Bounds}

We now derive some general bounds which we will use in the next section to analyze the asymptotic behavior of the buffer content distribution. We focus on a particular yet arbitrary source $i$ for which we assume $\rho_{i}<\gamma_{i i}$ to ensure stability. 
For any set $E \subseteq\{1, \ldots, N\}$, define

$$
\gamma_{i E}(\delta)=(1-\delta) \gamma_{i E}=(1-\delta) \rho_{i} \quad \text { for all } i \in S_{E}
$$

and

$$
\gamma_{i E}(\delta)=\phi_{i} R_{E}(\delta) \quad \text { for all } i \notin S_{E},
$$

with

$$
R_{E}(\delta)=\frac{1}{\sum_{j \notin S_{E}} \phi_{j}}\left(1-\sum_{j \in S_{E}} \gamma_{j E}(\delta)\right)=\frac{1}{\sum_{j \notin S_{E}} \phi_{j}}\left(1-(1-\delta) \sum_{j \in S_{E}} \rho_{j}\right)
$$

Notice that for $E \neq \emptyset, \sum_{i=1}^{N} \gamma_{i E}(\delta)=1$ for all values of $\delta$.

We first state a basic lemma which will play a central role in deriving the bounds.

Lemma 4.1 For any set $E \subseteq\{1, \ldots, N\}, S \supseteq S_{E}$,

$$
\sum_{j \in S} B_{j}(r, t) \geq \sum_{j \in S} \inf _{r \leq s \leq t}\left\{A_{j}(r, s)+\gamma_{j E}(\delta)(t-s)\right\},
$$

for all $\delta \geq \delta_{0}$ for some $\delta_{0}<0$.

\section{Proof}

The proof follows immediately from combining Lemma's C.1 and C.2.

We now present a lower bound for the buffer content distribution of source $i$. For any $j \neq i$, define $U_{i j}^{\delta}(r):=\sup _{s \geq r}\left\{\gamma_{j i}(\delta)(s-r)-A_{j}(r, s)\right\}$. For $\delta>0$ such that $\gamma_{i j}(\delta)<\rho_{j}$, let $\mathbf{U}_{i j}^{\delta}$ be a stochastic variable with as distribution the distribution of $U_{i j}^{\delta}(r)$ (which does not depend on $r$ ).

Lemma 4.2 (Lower bound) For $\delta>0$ sufficiently small,

$$
\mathbb{P}\left\{\mathbf{V}_{i}>x\right\} \geq \mathbb{P}\left\{\mathbf{V}_{i}^{\gamma_{i i}(\delta)}-\sum_{j \neq i} \mathbf{U}_{i j}^{\delta}>x\right\}
$$

\section{Proof}

Notice that $\sum_{j=1}^{N} B_{j}(r, t) \leq t-r$, so that $B_{i}(r, t) \leq t-r-\sum_{j \neq i} B_{j}(r, t)$ for all $0 \leq r \leq t$.

Thus, from (1), for any $0 \leq r \leq t$,

$$
V_{i}(t) \geq A_{i}(r, t)-(t-r)+\sum_{j \neq i} B_{j}(r, t) .
$$


By definition, $S_{i} \subseteq\{1, \ldots, N\} \backslash\{i\}$. Hence, from Lemma 4.1, for any $\delta \geq 0$,

$$
\sum_{j \neq i} B_{j}(r, t) \geq \sum_{j \neq i} \inf _{r \leq s \leq t}\left\{A_{j}(r, s)+\gamma_{j i}(\delta)(t-s)\right\}
$$

Plugging (4) into (3), for any $\delta \geq 0$ and $0 \leq r \leq t$,

$$
\begin{aligned}
V_{i}(t) & \geq A_{i}(r, t)-(t-r)+\sum_{j \neq i} \inf _{r \leq s \leq t}\left\{A_{j}(r, s)+\gamma_{j i}(\delta)(t-s)\right\} \\
& =A_{i}(r, t)-\gamma_{i i}(\delta)(t-r)-\sum_{j \neq i} \gamma_{j i}(\delta)(t-r)+\sum_{j \neq i} \inf _{r \leq s \leq t}\left\{A_{j}(r, s)+\gamma_{j i}(\delta)(t-s)\right\} \\
& \geq A_{i}(r, t)-\gamma_{i i}(\delta)(t-r)+\sum_{j \neq i} \inf _{s \geq r}\left\{A_{j}(r, s)-\gamma_{j i}(\delta)(s-r)\right\} \\
& =A_{i}(r, t)-\gamma_{i i}(\delta)(t-r)-\sum_{j \neq i} U_{i j}^{\delta}(r) .
\end{aligned}
$$

Denote $r^{*}:=\arg \sup _{0 \leq r \leq t}\left\{A_{i}(r, t)-\gamma_{i i}(\delta)(t-r)\right\}$, so that $V_{i}^{\gamma_{i i}(\delta)}(t)=A_{i}\left(r^{*}, t\right)-\gamma_{i i}(\delta)\left(t-r^{*}\right)$. Substituting $r=r^{*}$ in (5) then yields

$$
V_{i}(t) \geq V_{i}^{\gamma_{i i}(\delta)}(t)-\sum_{j \neq i} U_{i j}^{\delta}\left(r^{*}\right) .
$$

From the definition it is easily seen that for $\delta>0, \gamma_{j i}(\delta)<\gamma_{j i}=\rho_{j}$ for all $j \in S_{i}$, and $\gamma_{j i}(\delta)>\gamma_{j i}$ with $\gamma_{j i}(\delta) \downarrow \gamma_{j i}$ for $\delta \downarrow 0$ for all $j \notin S_{i}$. In particular, $\gamma_{i i}(\delta)>\rho_{i}$, because $\gamma_{i i}>\rho_{i}$. Since $\gamma_{j i}<\rho_{j}$ for $j \notin S_{i}, j \neq i$, we also have that for $\delta$ sufficiently small, $\gamma_{j i}(\delta)<\rho_{j}$ for $j \notin S_{i}$, $j \neq i$. Hence, for $\delta$ sufficiently small, $\gamma_{j i}(\delta)<\rho_{j}$ for all $j \neq i$, so that $\mathbf{U}_{i j}^{\delta}$ is well-defined.

Also, note that $r^{*}, V_{i}^{\gamma_{i i}(\delta)}(t)$ only depend on $A_{i}(s, t)$, and are independent of $U_{i j}^{\delta}(s), s \geq 0$ (fixed). Hence, for $\delta>0$ sufficiently small,

$$
\begin{aligned}
\mathbb{P}\left\{V_{i}(t)>x \mid r^{*}\right\} & \geq \mathbb{P}\left\{V_{i}^{\gamma_{i i}(\delta)}(t)-\sum_{j \neq i} U_{i j}^{\delta}\left(r^{*}\right)>x \mid r^{*}\right\} \\
& =\mathbb{P}\left\{V_{i}^{\gamma_{i i}(\delta)}(t)-\sum_{j \neq i} \mathbf{U}_{i j}^{\delta}>x \mid r^{*}\right\} .
\end{aligned}
$$

Thus, in the stationary regime, (2) holds.

We now present an upper bound for the buffer content distribution of source $i$.

Lemma 4.3 (Upper bound) For $\delta>0$ sufficiently small,

$$
\mathbb{P}\left\{\mathbf{V}_{i}>x\right\} \leq \mathbb{P}\left\{\mathbf{V}_{i}^{\gamma_{i E}(-\delta)}+\sum_{j \in S_{E}} \mathbf{V}_{j}^{(1+\delta) \rho_{j}}>x \text { for all sets } E \ni i \text { with } \gamma_{i E}>\rho_{i}\right\}
$$




\section{Proof}

Since source $i$ is stable, the distribution of $\mathbf{V}_{i}$ does not depend on the values of $V_{j}(0)$. Hence, we may assume $V_{j}(0)=0$ for all $j=1, \ldots, N$.

Then from (1),

$$
V_{i}(t) \leq \sum_{j \in S_{E} \cup\{i\}} V_{j}(t)=\sum_{j \in S_{E} \cup\{i\}}\left\{A_{j}(0, t)-B_{j}(0, t)\right\} .
$$

From Lemma 4.1 , for any $\delta \leq \delta_{0}$,

$$
\sum_{j \in S_{E} \cup\{i\}} B_{j}(0, t) \geq \sum_{j \in S_{E} \cup\{i\}} \inf _{0 \leq s \leq t}\left\{A_{j}(0, s)+\gamma_{j E}(-\delta)(t-s)\right\}
$$

for some $\delta_{0}>0$.

Substituting (8) into (7), for any $\delta \leq \delta_{0}$,

$$
\begin{aligned}
V_{i}(t) & \leq \sum_{j \in S_{E} \cup\{i\}}\left\{A_{j}(0, t)-\inf _{0 \leq s \leq t}\left\{A_{j}(0, s)+\gamma_{j E}(-\delta)(t-s)\right\}\right\} \\
& =\sum_{j \in S_{E} \cup\{i\}}\left\{A_{j}(0, t)+\sup _{0 \leq s \leq t}\left\{-A_{j}(0, s)-\gamma_{j E}(-\delta)(t-s)\right\}\right\} \\
& =\sum_{j \in S_{E} \cup\{i\}} \sup _{0 \leq s \leq t}\left\{A_{j}(s, t)-\gamma_{j E}(-\delta)(t-s)\right\} \\
& =\sum_{j \in S_{E} \cup\{i\}} V_{j}^{\gamma_{j E}(-\delta)}(t) \\
& =V_{i}^{\gamma_{i E}(-\delta)}(t)+\sum_{j \in S_{E}} V_{j}^{(1+\delta) \rho_{j}}(t)
\end{aligned}
$$

for all sets $E \ni i$ with $\gamma_{i E}>\rho_{i}$.

From the definition it is easily seen that for $\delta>0, \gamma_{i E}(-\delta)<\gamma_{i E}$ with $\gamma_{i E}(\delta) \uparrow \gamma_{i E}$ for $\delta \downarrow 0$. Since $\gamma_{i E}>\rho_{i}$, we have that $\gamma_{i E}(-\delta)>\rho_{i}$ for $\delta$ sufficiently small, and hence $\mathbf{V}_{i}^{\gamma_{i E}(-\delta)}$ is well-defined.

Thus, in the stationary regime, (6) holds for $\delta>0$ sufficiently small.

\section{Asymptotic behavior}

We now use the bounds from the previous section to determine the asymptotic behavior of the buffer content distribution. We consider a source $i$ which satisfies Properties 2.1, 2.2, and 2.3 with $\rho_{i}<\gamma_{i i}<\sigma_{i}$, and assume the following condition holds. 
Condition 1 For all sets $E \not \ngtr i$ with $\gamma_{i E \cup\{i\}} \leq \rho_{i}$,

$$
\lim _{x \rightarrow \infty} \frac{\prod_{j \in E} \bar{W}_{j}^{\rho_{j}(1+\delta)}(x)}{\bar{W}_{i}^{c}(x)}=0, \text { for any } \delta>0, c \in\left(\rho_{i}, \sigma_{i}\right) .
$$

Condition 1 postulates that the tail of $\min _{j \in E} \mathbf{V}_{j}^{\rho_{j}(1+\delta)}$ is lighter than the tail of $\mathbf{V}_{i}^{c}$ for all sets $E \not \supset i$ with $\gamma_{i E \cup\{i\}} \leq \rho_{i}$. In case of instantaneous arrivals, Theorem 2.1 then implies that the tail of $\min _{j \in E} \mathbf{B}_{j}^{r}$ must then be lighter than the tail of $\mathbf{B}_{i}^{r}$. Similarly, in case of On/Off processes, Theorem 2.2 indicates that the tail of $\min _{j \in E} \mathbf{A}_{j}^{r}$ must then be dominated by the tail of $\mathbf{A}_{i}^{r}$. The inequality $\gamma_{i E \cup\{i\}} \leq \rho_{i}$ means that source $i$ could be pushed into instability if the sources $j \in E$ continuously claimed their full share of the capacity. Thus, Condition 1 guarantees that only sources with combined lighter tails could potentially drive source $i$ into instability. In other words, sources with combined heavier tails cannot drive source $i$ into instability.

As a special but important case, consider a scenario where some of the sources have regularly varying tails, while the others have exponential tails. Specifically, suppose that $\bar{W}_{i}^{c}(\cdot)$ is regularly varying with index $1-\nu_{i}$ for the sources $i \in R, R \subseteq\{1, \ldots, N\}$. For the other sources $j \notin R, \bar{W}_{j}^{c}(x)=o\left(x^{-\zeta}\right)$ for any $c>\rho_{j}$ and some $\zeta>0$. In this case, for the sources $i \in R$, Condition 1 may be rewritten as follows.

\section{Condition 1'}

For all sets $E \subseteq R, E \not \supset i$, with $\gamma_{i E} \leq \rho_{i}, \sum_{j \in E}\left(\nu_{j}-1\right)>\nu_{i}-1$.

We now state the main theorem of the paper.

Theorem 5.1 Consider a source $i$ which satisfies Properties 2.1, 2.2, and 2.3 with $\rho_{i}<\gamma_{i i}<$ $\sigma_{i}$. If Condition 1 holds, then

$$
\mathbb{P}\left\{\mathbf{V}_{i}>x\right\} \sim \mathbb{P}\left\{\mathbf{V}_{i}^{\gamma_{i i}}>x\right\} \quad \text { as } \quad x \rightarrow \infty
$$

Before giving the formal proof of Theorem 5.1, we first provide an intuitive interpretation and discuss the significance of Condition 1.

The result shows that an individual source with long-tailed traffic characteristics is effectively served at constant rate $\gamma_{i i}$. Remember $\gamma_{i i}$ is the mean service rate that source $i$ would receive if it continuously claimed capacity. This suggests that the most likely scenario for source $i$ to build a large queue is to generate a large burst, or to experience a long On-period, while the other sources show average behavior. During that congestion period, source $i$ then receives service approximately at rate $\gamma_{i i}$.

If Condition 1 does not hold, then there is some set $E \not \ngtr i$ with heavier combined tails than source $i$ and $\gamma_{i E} \leq \rho_{i}$. We conjecture that the tail behavior of $\mathbf{V}_{i}$ in that case is determined 
by the set $E^{*}$ with the heaviest tails, i.e., $\lim _{x \rightarrow \infty} \prod_{j \in E} \bar{W}_{j}^{\rho_{j}(1+\delta)}(x) / \prod_{j \in E^{*}} \bar{W}_{j}^{\rho_{j}(1+\delta)}(x)=0$ for all $E \neq E^{*}$ with $\gamma_{i E} \leq \rho_{i}$. Observe that the tail of $\mathbf{V}_{i}$ is now heavier than when source $i$ were served in isolation at a stable rate. The most likely scenario for source $i$ to build a large queue is that the sources $j \in E^{*}$ generate large bursts, or experience long On-periods, while the other sources, including source $i$, show average behavior. Source $i$ then receives service approximately at rate $\gamma_{i E^{*}} \leq \rho_{i}$, so that the queue will roughly grow at rate $\rho_{i}-\gamma_{i E^{*}}$ for a significant period of time.

The conjecture has recently been proved in [9] for the case of $N=2$ sources and a class of arrival processes which include regularly varying burst sizes, i.e., $1-B_{i}(x) \sim l_{i}(x) x^{-\nu_{i}}$ as $x \rightarrow \infty$, with $\nu_{i}>1$ and $l_{i}(\cdot)$ slowly varying functions, $i=1,2$, see the definition in Appendix A. Suppose that $\rho_{1}>\phi_{1}, \rho_{1}+\rho_{2}<1$, so that $\rho_{2}<\phi_{2}$. Then $\gamma_{11}=1-\rho_{2}>\rho_{1}, \gamma_{22}=\phi_{2}>\rho_{2}$, $\gamma_{1\{1,2\}}=\phi_{1}<\rho_{1}$, and $\gamma_{2\{1,2\}}=\phi_{2}>\rho_{2}$. Thus Condition 1 holds for source 2 , so that if source 2 satisfies Properties 2.1, 2.2, and 2.3, then $\mathbb{P}\left\{\mathbf{V}_{2}>x\right\} \sim \mathbb{P}\left\{\mathbf{V}_{2}^{\phi_{2}}>x\right\}$ as $x \rightarrow \infty$. Now suppose that $\nu_{1}>\nu_{2}$. Then Condition 1 does not hold for source 1 , and source 1 inherits the tail behavior of source 2, regardless of whether or not it satisfies Properties 2.1, 2.2, and 2.3, and thus experiences heavier tail behavior than when served in isolation. This may be explained as follows. Note that when source 2 is backlogged, source 1 receives service at rate $\phi_{1}$, so queue 1 will roughly grow at rate $\rho_{1}-\phi_{1}$. When source 2 is not backlogged, queue 1 will drain at rate $1-\rho_{1}$. Thus the tail behavior of source 1 is equivalent to the tail behavior of a single On/Off source served at constant rate $1-\rho_{1}$, with peak rate $1-\phi_{1}$, as On-period distribution the busy-period distribution of source 2 (which is also regularly varying of index $\nu_{2}$ ), and fraction Off-time $1-\rho_{2} / \phi_{2}$. This is also shown for a closely related coupled-processors model in [8].

A special but important case is a scenario where $\rho_{i}<\phi_{i}$ for all $i=1, \ldots, N$. It is easily verified that in that case $\gamma_{i i}=1-\sum_{j \neq i} \rho_{j}>1-\sum_{j \neq i} \phi_{j}=\phi_{i}>\rho_{i}$. Also, for all sets $E \not \supset i, \gamma_{i E \cup\{i\}}=\frac{\phi_{i}}{\phi_{i}+\sum_{j \in E} \phi_{j}}\left(1-\sum_{k \notin E} \rho_{k}+\rho_{i}\right)>\frac{\phi_{i}}{\phi_{i}+\sum_{j \in E} \phi_{j}}\left(1-\sum_{k \notin E} \phi_{k}+\phi_{i}\right)=\phi_{i}>\rho_{i}$. Thus, Condition 1 holds, so that Theorem 5.1 gives $\mathbb{P}\left\{\mathbf{V}_{i}>x\right\} \sim \mathbb{P}\left\{\mathbf{V}_{i}^{1-\sum_{j \neq i} \rho_{j}}>x\right\}$ as $x \rightarrow \infty$. This result can in fact be obtained using a simpler proof technique and under slightly weaker conditions, see [7] and also Remark 5.1 below.

Now suppose each of the sources were served in isolation. Then the required capacity to achieve similar tail behavior is $\sum_{i=1}^{N} \gamma_{i i}=\sum_{i=1}^{N}\left(1-\sum_{j \neq i} \rho_{j}\right)=\sum_{i=1}^{N}\left(1-\rho+\rho_{i}\right)=1+(N-1)(1-\rho)$. The latter quantity may typically be expected to be significantly larger than 1 . This suggests that GPS is effective in extracting high multiplexing gains, while protecting individual connections. 
Recall that besides stability, i.e., $\rho_{i}<\gamma_{i i}$, we also assume $\sigma_{i}>\gamma_{i i}$ in Theorem 5.1. In case of instantaneous arrivals, this assumption is not restrictive. However, in case of On/Off processes, $\sigma_{i}<r_{i}$, so that Theorem 5.1 does not apply when $r_{i} \leq \gamma_{i i}$.

If Condition 1 does not hold, then we expect the tail behavior of $\mathbf{V}_{i}$ in that case is still determined by the set $E^{*}$ as described above. If Condition 1 does hold, however, then we conjecture that, possibly under certain additional conditions, the tail behavior is determined by the set $E^{*}$ with the heaviest tails for which either (i) $\gamma_{i E}<\rho_{i}$, if $i \notin E$ or (ii) $\gamma_{i E}<r_{i}$, if $i \in E$. Observe that the tail of $\mathbf{V}_{i}$ is now lighter than when source $i$ were served in isolation. The most likely scenario for source $i$ to build a large queue is still that the sources $j \in E^{*}$ generate large bursts or experience long On-periods, while the other sources show average behavior.

We now give the proof of Theorem 5.1.

\section{Proof}

(Lower bound) From Lemma 4.2, for $\delta>0$ sufficiently small and any value of $y$, using independence,

$$
\begin{aligned}
\mathbb{P}\left\{\mathbf{V}_{i}>x\right\} & \geq \mathbb{P}\left\{\mathbf{V}_{i}^{\gamma_{i i}(\delta)}-\sum_{j \neq i} \mathbf{U}_{i j}^{\delta}>x\right\} \\
& \geq \mathbb{P}\left\{\mathbf{V}_{i}^{\gamma_{i i}(\delta)}>x+y, \sum_{j \neq i} \mathbf{U}_{i j}^{\delta} \leq y\right\} \\
& =\mathbb{P}\left\{\mathbf{V}_{i}^{\gamma_{i i}(\delta)}>x+y\right\} \mathbb{P}\left\{\sum_{j \neq i} \mathbf{U}_{i j}^{\delta} \leq y\right\}
\end{aligned}
$$

Thus,

$$
\frac{\mathbb{P}\left\{\mathbf{V}_{i}>x\right\}}{\mathbb{P}\left\{\mathbf{V}_{i}^{\gamma_{i i}}>x\right\}} \geq \frac{\mathbb{P}\left\{\mathbf{V}_{i}^{\gamma_{i i}(\delta)}>x+y\right\}}{\mathbb{P}\left\{\mathbf{V}_{i}^{\gamma_{i i}(\delta)}>x\right\}} \frac{\mathbb{P}\left\{\mathbf{V}_{i}^{\gamma_{i i}(\delta)}>x\right\}}{\mathbb{P}\left\{\mathbf{V}_{i}^{\gamma_{i i}}>x\right\}} \mathbb{P}\left\{\sum_{j \neq i} \mathbf{U}_{i j}^{\delta} \leq y\right\} .
$$

Using the fact that $\bar{W}_{i}^{c}(\cdot)$ satisfies Properties 2.1 and 2.3 for $c \in\left(\rho_{i}, \sigma_{i}\right)$,

$$
\begin{aligned}
\liminf _{x \rightarrow \infty} \frac{\mathbb{P}\left\{\mathbf{V}_{i}>x\right\}}{\mathbb{P}\left\{\mathbf{V}_{i}^{\gamma_{i i}}>x\right\}} & \geq \liminf _{x \rightarrow \infty} \frac{\mathbb{P}\left\{\mathbf{V}_{i}^{\gamma_{i i}(\delta)}>x\right\}}{\mathbb{P}\left\{\mathbf{V}_{i}^{\gamma_{i i}}>x\right\}} \mathbb{P}\left\{\sum_{j \neq i} \mathbf{U}_{i j}^{\delta} \leq y\right\} \\
& =\mathbb{P}\left\{\sum_{j \neq i} \mathbf{U}_{i j}^{\delta} \leq y\right\} / \limsup _{x \rightarrow \infty} \frac{\mathbb{P}\left\{\mathbf{V}_{i}^{\gamma_{i i}}>x\right\}}{\mathbb{P}\left\{\mathbf{V}_{i}^{\gamma_{i i}(\delta)}>x\right\}} \\
& =\mathbb{P}\left\{\sum_{j \neq i} \mathbf{U}_{i j}^{\delta} \leq y\right\} / H_{i}^{\gamma_{i i}(\delta)}\left(\gamma_{i i}(\delta)-\gamma_{i i}\right)
\end{aligned}
$$

for $\delta$ sufficiently small. 
Letting $y \rightarrow \infty$ and $\delta \downarrow 0$, observing that $\lim _{\delta \downarrow 0} \gamma_{i i}(\delta)=\gamma_{i i}$,

$$
\liminf _{x \rightarrow \infty} \frac{\mathbb{P}\left\{\mathbf{V}_{i}>x\right\}}{\mathbb{P}\left\{\mathbf{V}_{i}^{\gamma_{i i}}>x\right\}} \geq 1
$$

(Upper bound) Let us index the sets $E \ni i$ for which $\gamma_{i E}>\rho_{i}$ as $E_{0}, E_{1}, \ldots, E_{M}$.

Because $\rho_{i}<\gamma_{i i}$, we may assume $E_{0}=\{i\}$.

Hence, from Lemma 4.3, for $\delta>0$ sufficiently small and any value of $y$, using independence,

$$
\begin{aligned}
\mathbb{P}\left\{\mathbf{V}_{i}>x\right\} \leq & \mathbb{P}\left\{\mathbf{V}_{i}^{\gamma_{i E}(-\delta)}+\sum_{j \in S_{E}} \mathbf{V}_{j}^{(1+\delta) \rho_{j}}>x \text { for all sets } E \ni i \text { with } \gamma_{i E}>\rho_{i}\right\} \\
= & \mathbb{P}\left\{\mathbf{V}_{i}^{\gamma_{i i}(-\delta)}+\sum_{j \in S_{i}} \mathbf{V}_{j}^{(1+\delta) \rho_{j}}>x, \mathbf{V}_{i}^{\gamma_{i E_{m}}(-\delta)}+\sum_{j \in S_{E_{m}}} \mathbf{V}_{j}^{(1+\delta) \rho_{j}}>x \forall m=1, \ldots, M\right\} \\
\leq & \mathbb{P}\left\{\mathbf{V}_{i}^{\gamma_{i i}(-\delta)}>x-y \text { or } \sum_{j \in S_{i}} \mathbf{V}_{j}^{(1+\delta) \rho_{j}}>y,\right. \\
& \left.\mathbf{V}_{i}^{\gamma_{i E_{m}}(-\delta)}>x / N \text { or } \exists j_{m} \in S_{E_{m}}: \mathbf{V}_{j_{m}}^{(1+\delta) \rho_{j m}}>x / N \forall m=1, \ldots, M\right\} \\
\leq & \mathbb{P}\left\{\mathbf{V}_{i}^{\gamma_{i i}(-\delta)}>x-y\right\}+\mathbb{P}\left\{\sum_{j \in S_{i}} \mathbf{V}_{j}^{(1+\delta) \rho_{j}}>y, \exists m: \mathbf{V}_{i}^{\gamma_{i E m}(-\delta)}>x / N\right\} \\
+ & \sum_{j_{1} \in S_{E_{1}}, \ldots, j_{M} \in S_{E_{M}}} \mathbb{P}\left\{\mathbf{V}_{j_{m}}^{(1+\delta) \rho_{j_{m}}}>x / N \forall m=1, \ldots, M\right\} \\
\leq & \mathbb{P}\left\{\mathbf{V}_{i}^{\gamma_{i i}(-\delta)}>x-y\right\}+\mathbb{P}\left\{\sum_{j \in S_{i}} \mathbf{V}_{j}^{(1+\delta) \rho_{j}}>y\right\} \sum_{m=1}^{M} \mathbb{P}\left\{\mathbf{V}_{i}^{\gamma_{i E}(-\delta)}>x / N\right\} \\
+ & \sum_{j_{1} \in S_{E_{1}, \ldots, j_{M} \in S_{E_{M}}} \prod_{j \in\left\{j_{1}, \ldots, j_{M}\right\}} \mathbb{P}\left\{\mathbf{V}_{j}^{(1+\delta) \rho_{j}}>x / N\right\} .}
\end{aligned}
$$

Thus,

$$
\begin{aligned}
\frac{\mathbb{P}\left\{\mathbf{V}_{i}>x\right\}}{\mathbb{P}\left\{\mathbf{V}_{i}^{\gamma_{i i}}>x\right\}} \leq \frac{\mathbb{P}\left\{\mathbf{V}_{i}^{\gamma_{i i}(-\delta)}>x-y\right\}}{\mathbb{P}\left\{\mathbf{V}_{i}^{\gamma_{i i}(-\delta)}>x\right\}} \frac{\mathbb{P}\left\{\mathbf{V}_{i}^{\gamma_{i i}(-\delta)}>x\right\}}{\mathbb{P}\left\{\mathbf{V}_{i}^{\gamma_{i i}}>x\right\}} \\
+\mathbb{P}\left\{\sum_{j \in S_{i}} \mathbf{V}_{j}^{(1+\delta) \rho_{j}}>y\right\} \sum_{m=1}^{M} \frac{\mathbb{P}\left\{\mathbf{V}_{i}^{\gamma_{i E}}(-\delta)\right.}{\mathbb{P}\left\{\mathbf{V}_{i}^{\gamma_{i i}}>x / N\right\}} \frac{\mathbb{P}\left\{\mathbf{V}_{i}^{\gamma_{i i}}>x / N\right\}}{\mathbb{P}\left\{\mathbf{V}_{i}^{\gamma_{i i}}>x\right\}} \\
+\sum_{j_{1} \in S_{E_{1}}, \ldots, j_{M} \in S_{E_{M}}} \frac{\prod_{j \in\left\{j_{1}, \ldots, j_{M}\right\}} \mathbb{P}\left\{\mathbf{V}_{j}^{(1+\delta) \rho_{j}}>x / N\right\}}{\mathbb{P}\left\{\mathbf{V}_{i}^{\gamma_{i i}}>x / N\right\}} \frac{\mathbb{P}\left\{\mathbf{V}_{i}^{\gamma_{i i}}>x / N\right\}}{\mathbb{P}\left\{\mathbf{V}_{i}^{\gamma_{i i}}>x\right\}}
\end{aligned}
$$

Using the fact that $\bar{W}_{i}^{c}(\cdot)$ satisfies Properties $2.1,2.2$, and 2.3 for $c \in\left(\rho_{i}, \sigma_{i}\right)$,

$$
\begin{aligned}
\limsup _{x \rightarrow \infty} \frac{\mathbb{P}\left\{\mathbf{V}_{i}>x\right\}}{\mathbb{P}\left\{\mathbf{V}_{i}^{\gamma_{i i}}>x\right\}} & \leq H_{i}^{\gamma_{i i}}\left(\gamma_{i i}-\gamma_{i i}(-\delta)\right) \\
& +\mathbb{P}\left\{\sum_{j \in S_{i}} \mathbf{V}_{j}^{(1+\delta) \rho_{j}}>y\right\} G_{i}^{\gamma_{i i}}(1 / N) \sum_{m=1}^{M} H_{i}^{\gamma_{i i}}\left(\gamma_{i i}-\gamma_{i E_{m}}(-\delta)\right)
\end{aligned}
$$




$$
+G_{i}^{\gamma_{i i}}(1 / N) \sum_{j_{1} \in S_{E_{1}}, \ldots, j_{M} \in S_{E_{M}}} \limsup _{x \rightarrow \infty} \frac{\prod_{j \in\left\{j_{1}, \ldots, j_{M}\right\}} \mathbb{P}\left\{\mathbf{V}_{j}^{(1+\delta) \rho_{j}}>x / N\right\}}{\mathbb{P}\left\{\mathbf{V}_{i}^{\gamma_{i i}}>x / N\right\}}
$$

Now consider a set $\left\{j_{1}, \ldots, j_{M}\right\}$ with $j_{1} \in S_{E_{1}}, \ldots, j_{M} \in S_{E_{M}}$. By definition, $j_{1} \notin E_{1}, \ldots, j_{M} \notin$ $E_{M}$, so that $\left\{i, j_{1}, \ldots, j_{M}\right\} \neq E_{1}, \ldots, E_{M}$. Consequently, $\gamma_{i\left\{i, j_{1}, \ldots, j_{M}\right\}} \leq \rho_{i}$. Condition 1 then implies that

$$
\limsup _{x \rightarrow \infty} \frac{\prod_{j \in\left\{j_{1}, \ldots, j_{M}\right\}} \mathbb{P}\left\{\mathbf{V}_{j}^{(1+\delta) \rho_{j}}>x / N\right\}}{\mathbb{P}\left\{\mathbf{V}_{i}^{\gamma_{i i}}>x / N\right\}}=0 .
$$

Hence,

$$
\begin{aligned}
\limsup _{x \rightarrow \infty} \frac{\mathbb{P}\left\{\mathbf{V}_{i}>x\right\}}{\mathbb{P}\left\{\mathbf{V}_{i}^{\gamma_{i i}}>x\right\}} & \leq H_{i}^{\gamma_{i i}}\left(\gamma_{i i}-\gamma_{i i}(-\delta)\right) \\
& +\mathbb{P}\left\{\sum_{j \in S_{i}} \mathbf{V}_{j}^{(1+\delta) \rho_{j}}>y\right\} G_{i}^{\gamma_{i i}}(1 / N) \sum_{m=1}^{M} H_{i}^{\gamma_{i i}}\left(\gamma_{i i}-\gamma_{i E_{m}}(-\delta)\right) .
\end{aligned}
$$

Letting $y \rightarrow \infty$ and $\delta \downarrow 0$, observing that $\lim _{\delta \downarrow 0} \gamma_{i i}(-\delta)=\gamma_{i i}$,

$$
\limsup _{x \rightarrow \infty} \frac{\mathbb{P}\left\{\mathbf{V}_{i}>x\right\}}{\mathbb{P}\left\{\mathbf{V}_{i}^{\gamma_{i i}}>x\right\}} \leq 1
$$

Remark 5.1 Notice that neither Property 2.2 nor Condition 1 were actually used in establishing the lower bound in the proof of Theorem 5.1. Now suppose that there is a set $E \ni i$ such that $\gamma_{i E}>\rho_{i}$ and for any $c \in\left(\rho_{i}, \sigma_{i}\right), \lim _{x \rightarrow \infty} \bar{W}_{j}^{(1+\delta) \rho_{j}}(x) / \bar{W}_{i}^{c}(x)=0$ for all $j \in S_{E}$. In that case, Property 2.2 and Condition 1 are not needed in obtaining the upper bound either, so that Properties 2.1 and 2.3 are sufficient for Theorem 5.1 to hold. Two extreme cases where there is such a set $E \ni i$ are: (i) $\rho_{i}<\phi_{i}$ with $E=\{1, \ldots, N\}$; (ii) $\lim _{x \rightarrow \infty} W_{j}^{(1+\delta) \rho_{j}}(x) / W_{i}^{c}(x)=0$ for all $j \in S_{i}$ with $E=\{i\}$.

\section{Conclusion}

We analyzed the asymptotic behavior of long-tailed traffic sources under the Generalized Processor Sharing (GPS) discipline. GPS-based scheduling algorithms, such as Weighted Fair Queueing, have emerged as an important mechanism for achieving differentiated quality-ofservice in integrated-services networks.

Under certain conditions, we proved that in an asymptotic sense an individual source with longtailed traffic characteristics is effectively served at a constant rate, which may be interpreted 
as the maximum feasible average rate for that source to be stable. Thus, asymptotically, the source is only affected by the traffic characteristics of the other sources through their average rate. In particular, the source is essentially immune from excessive activity of sources with 'heavier'-tailed traffic characteristics. This suggests that GPS-based scheduling algorithms provide an effective mechanism for extracting high multiplexing gains, while protecting individual connections.

Acknowledgment The authors gratefully acknowledge a useful suggestion made by Bert Zwart.

\section{References}

[1] Abate, J., Whitt, W. (1997). Asymptotics for M/G/1 low-priority waiting-time tail probabilities. Queueing Systems 25, 173-233.

[2] Agrawal, R., Makowski, A.M., Nain, Ph. (1999). On a reduced load equivalence for a fluid model under subexponential assumptions. Queueing Systems, to appear.

[3] Anantharam, V. (1997). Scheduling strategies and long-range dependence. Technical report, University of California, Berkeley.

[4] Asmussen, S., Schmidli, H., Schmidt, V. (1999). Tail probabilities for non-standard risk and queueing processes with subexponential jumps. Adv. Appl. Prob. 31, 422-447.

[5] Bertsimas, D., Paschalidis, I.Ch., Tsitsiklis, J.N. (1997). Large deviations analysis of the generalized processor sharing policy. Report Boston University.

[6] Bingham, N.H., Goldie, C.M., Teugels, J.L. (1987). Regular Variation (Cambridge University Press, Cambridge, UK).

[7] Borst, S.C., Boxma, O.J., Jelenković, P.R. (1999). Generalized processor sharing with long-tailed traffic sources. In: Teletraffic Engineering in a Competitive World, Proc. ITC16, Edinburgh, UK, eds. P. Key, D. Smith (North-Holland, Amsterdam), 345-354.

[8] Borst, S.C., Boxma, O.J., Jelenković, P.R. (2000). Coupled processors with regularly varying service times. In: Proc. Infocom 2000, to appear

[9] Borst, S.C., Boxma, O.J., Jelenković, P.R. (1999). Induced burstiness in generalized processor sharing queues with long-tailed traffic flows. In: Proc. 37th Annual Allerton Conference on Communication, Control, and Computing.

[10] Boxma, O.J. (1996). Fluid queues and regular variation. Perf. Eval. 27 \& 28, 699-712. 
[11] Boxma, O.J. (1997). Regular variation in a multi-source fluid queue. In: Teletraffic Contributions for the Information Age, Proc. ITC-15, Washington DC, eds. V. Ramaswami, P.E. Wirth (North-Holland, Amsterdam), 391-402.

[12] Boxma, O.J., Cohen, J.W. (1998). The single server queue: Heavy tails and heavy traffic. In: Self-similar Network Traffic and Performance Evaluation, eds. K. Park, W. Willinger (Wiley, New York), to appear.

[13] Boxma, O.J., Cohen, J.W., Deng, Q. (1999). Heavy-traffic analysis of the M/G/1 queue with priority classes. In: Teletraffic Engineering in a Competitive World, Proc. ITC-16, Edinburgh, UK, eds. P. Key, D. Smith (North-Holland, Amsterdam), 1157-1167.

[14] Boxma, O.J. Dumas, V. (1998). Fluid queues with heavy-tailed activity period distributions. Computer Communications 21, 1509-1529.

[15] Chistyakov, V.P. (1964). A theorem on sums of independent positive random variables and its application to branching random processes. Theory Prob. Appl. 9, 640-648.

[16] Choudhury, G.L., Whitt, W. (1997). Long-tail buffer-content distributions in broadband networks. Perf. Eval. 30, 177-190.

[17] Cline, D.B.H. (1994). Intermediate regular and $\pi$ variation. In: Proc. London Math. Soc. 68, 594-616.

[18] Cohen, J.W. (1973). Some results on regular variation for distributions in queueing and fluctuation theory. J. Appl. Prob. 10, 343-353.

[19] Dupuis, P., Ramanan, K. (1998). A Skorokhod Problem formulation and large deviation analysis of a processor sharing model. Queueing Systems 28, 109-124.

[20] Goldie, C.M., Klüppelberg, C. (1997). Subexponential distributions. In: A Practical Guide to Heavy Tails: Statistical Techniques and Applications, eds. R.J. Adler, R.E Feldman, M.S. Taqqu (Birkhäuser), 435-459.

[21] Heyman, D.P, Lakshman, T.V. (1996). Source models for VBR broadcast-video traffic. IEEE/ACM Trans. Netw. 4, 40-48.

[22] Heath, D., Resnick, S., Samorodnitsky, G. (1998). Heavy tails and long range dependence in on/off processes and associated fluid models. Math. Oper. Res. 23, 145-165.

[23] Jelenković, P.R. (1998). Asymptotic results for queues with subexponential arrival processes. In: Self-similar Network Traffic and Performance Evaluation, eds. K. Park, W. Willinger (Wiley, New York), to appear. 
[24] Jelenković, P.R., Lazar., A.A. (1999). Asymptotic results for multiplexing subexponential on-off processes. Adv. Appl. Prob. 31, 394-421.

[25] Jelenković, P.R., Lazar, A.A. (1998). Subexponential asymptotics of a Markov-modulated random walk with queueing applications. J. Appl. Prob. 35, 325-347.

[26] Jelenković, P.R., Lazar, A.A., Semret, N. (1997). The effect of multiple time scales and subexponentiality of MPEG video streams on queueing behavior. IEEE J. Sel. Areas Commun. 15, 1052-1071.

[27] Karamata, J. (1930). Sur un mode de croissance régulière des fonctions. Mathematica (Cluj) 4, 38-53.

[28] Klüppelberg, C. (1988). Subexponential distributions and integrated tails. J. Appl. Prob. 25, 132-141.

[29] Leland, W.E., Taqqu, M.S., Willinger, W., Wilson, D.V. (1993). On the self-similar nature of Ethernet traffic. In: Proc. SIGCOMM '93, 183-193.

[30] Massoulié, L. (1998). Large deviations for polling and Weighted Fair Queueing service systems. Report France Télécom-CNET.

[31] Pakes, A.G. (1975). On the tails of waiting-time distributions. J. Appl. Prob. 12, 555-564.

[32] Parekh, A.K., Gallager, R.G. (1993). A generalized processor sharing approach to flow control in integrated services networks: the single-node case. IEEE/ACM Trans. Netw. 1, 344-357.

[33] Parekh, A.K., Gallager, R.G. (1994). A generalized processor sharing approach to flow control in integrated services networks: the multiple node case. IEEE/ACM Trans. Netw. 2, $137-150$.

[34] Rolski, T., Schlegel, S., Schmidt, V. (1999). Asymptotics of Palm-stationary buffer content distributions in fluid flow queues. Adv. Appl. Prob. 31, 235-253.

[35] Veraverbeke, N. (1977). Asymptotic behaviour of Wiener-Hopf factors of a random walk. Stoch. Proc. Appl. 5, 27-37.

[36] Zhang, Z.-L. (1998). Large deviations and the generalized processor sharing scheduling for a multiple-queue system. Queueing Systems 28, 349-376.

[37] Zhang, Z.-L., Towsley, D., Kurose, J. (1995). Statistical analysis of the generalized processor sharing discipline. IEEE J. Sel. Areas Commun. 13, 1071-1080. 
[38] Zwart, A.P. (1999). Sojourn times in a multiclass processor sharing queue. In: Teletraffic Engineering in a Competitive World, Proc. ITC-16, Edinburgh, UK, eds. P. Key, D. Smith (North-Holland, Amsterdam), 335-344.

[39] Zwart, A.P., Boxma, O.J. (1999). Sojourn time asymptotics in the M/G/1 processor sharing queue. Queueing Systems, to appear.

\section{A Definitions}

Definition 1 A distribution function $F(\cdot)$ on $[0, \infty)$ is called long-tailed $(F(\cdot) \in \mathcal{L})$ if

$$
\lim _{x \rightarrow \infty} \frac{1-F(x-y)}{1-F(x)}=1, \text { for all real } y \text {. }
$$

Definition 2 A distribution function $F(\cdot)$ on $[0, \infty)$ is called subexponential $(F(\cdot) \in \mathcal{S})$ if

$$
\lim _{x \rightarrow \infty} \frac{1-F^{2 *}(x)}{1-F(x)}=2,
$$

where $F^{2 *}(\cdot)$ is the 2 -fold convolution of $F(\cdot)$ with itself, i.e., $F^{2 *}(x)=\int_{0}^{x} F(x-y) F(\mathrm{~d} y)$.

The class of subexponential distributions was introduced by Chistyakov [15]. The definition is motivated by the simplification of the asymptotic analysis of the convolution tails. A wellknown subclass of $\mathcal{S}$ is the class $\mathcal{R}$ of regularly-varying distributions (which contains the Pareto distribution):

Definition 3 A distribution function $F(\cdot)$ on $[0, \infty)$ is called regularly varying of index $-\nu$ $\left(F(\cdot) \in \mathcal{R}_{-\nu}\right)$ if

$$
F(x)=1-\frac{l(x)}{x^{\nu}}, \quad \nu \geq 0
$$

where $l: \mathbb{R}_{+} \rightarrow \mathbb{R}_{+}$is a function of slow variation, i.e., $\lim _{x \rightarrow \infty} l(\eta x) / l(x)=1, \eta>1$.

The class of regularly-varying functions was introduced by Karamata [27]; a key reference is Bingham et al. [6]. It is easily seen that $\mathcal{R} \subset \mathcal{S} \subset \mathcal{L}$. Examples of subexponential distributions which do not belong to $\mathcal{R}$ include the Weibull, lognormal, and Benktander distributions (see Klüppelberg [28]). A useful extension of $\mathcal{R}$ is the class $\mathcal{I} \mathcal{R}$ of intermediately regularly-varying distributions:

Definition 4 A distribution function $F(\cdot)$ on $[0, \infty)$ is called intermediately regularly varying $(F(\cdot) \in \mathcal{I R})$ if

$$
\lim _{\eta \uparrow 1} \limsup _{x \rightarrow \infty} \frac{1-F(\eta x)}{1-F(x)}=1 .
$$


A further extension is the class $\mathcal{D} \mathcal{R}$ of dominatedly varying distributions (see Cline [17]; $\mathcal{R} \subset$ $\mathcal{I R} \subset(\mathcal{D R} \cap \mathcal{L}) \subset \mathcal{S})$

Definition 5 A distribution function $F(\cdot)$ on $[0, \infty)$ is called dominatedly varying $(F(\cdot) \in \mathcal{D} \mathcal{R})$ if

$$
\limsup _{x \rightarrow \infty} \frac{1-F(\eta x)}{1-F(x)}<\infty, \text { for some real } \eta \in(0,1)
$$

\section{B Stability issues}

We now identify which sources are stable and which ones are unstable. Source $i$ is considered 'stable' if the mean service rate is $\rho_{i}$. For the ease of presentation, we assume the sources are indexed such that

$$
\frac{\rho_{1}}{\phi_{1}} \leq \ldots \leq \frac{\rho_{N}}{\phi_{N}}
$$

Define $S$ as the set of stable sources. Denote by $\gamma_{i}$ the mean service rate for source $i$ (assuming it exists).

We have $\gamma_{i} \leq \rho_{i}$ for all $i=1, \ldots, N$, with equality for all $i \in S$. Also, if $j \notin S$, then $\frac{\gamma_{i}}{\phi_{i}} \leq \frac{\gamma_{j}}{\phi_{j}}$ for all $i=1, \ldots, N$.

In particular, we have $\frac{\gamma_{i}}{\phi_{i}}=\frac{\gamma_{j}}{\phi_{j}}$ for any pair of sources $i, j \notin S$, so $\gamma_{i}=\phi_{i} R$ for all $i \notin S$ for some $R \geq 1$. To determine $R$, observe that $\sum_{i=1}^{N} \gamma_{i}=1$ if $S \neq\{1, \ldots, N\}$, which gives

$$
R=\frac{1}{\sum_{j \notin S} \phi_{j}}\left(1-\sum_{j \in S} \rho_{j}\right) .
$$

We first prove a lemma that characterizes the structure of the set $S$.

Lemma B.1 With the above ordering of the sources, the set $S$ is of the form $\{1, \ldots, K\}$ for some $K$.

\section{Proof}

Suppose not, i.e., there are sources $i$ and $j$, with $i<j, i \notin S$, and $j \in S$. Then we have $\gamma_{i}<\rho_{i}$, $\gamma_{j}=\rho_{j}$, and $\frac{\gamma_{i}}{\phi_{i}} \geq \frac{\gamma_{j}}{\phi_{j}}$. Thus, $\frac{\rho_{i}}{\phi_{i}}>\frac{\rho_{j}}{\phi_{j}}$, which would contradict the ordering of the sources.

We now prove an auxiliary lemma. 
Lemma B.2 With the above ordering of the sources, if

$$
\rho_{k}>\frac{\phi_{k}}{\sum_{j=k}^{N} \phi_{j}}\left(1-\sum_{j=1}^{k-1} \rho_{j}\right)
$$

then

$$
\rho_{k+1}>\frac{\phi_{k+1}}{\sum_{j=k+1}^{N} \phi_{j}}\left(1-\sum_{j=1}^{k} \rho_{j}\right)
$$

\section{Proof}

First observe the equivalence relation

$$
\rho_{k}>\frac{\phi_{k}}{\sum_{j=k}^{N} \phi_{j}}\left(1-\sum_{j=1}^{k-1} \rho_{j}\right) \Longleftrightarrow \rho_{k}>\frac{\phi_{k}}{\sum_{j=k+1}^{N} \phi_{j}}\left(1-\sum_{j=1}^{k} \rho_{j}\right) .
$$

The proof then immediately follows from the fact that $\frac{\rho_{k}}{\phi_{k}} \leq \frac{\rho_{k+1}}{\phi_{k+1}}$.

The next proposition now identifies the set of stable sources.

\section{Proposition 3.1}

With the above ordering of the sources, the set of stable sources is $S=\left\{1, \ldots, K^{*}\right\}$, with

$$
K^{*}=\max _{k=1, \ldots, N}\left\{k: \frac{\rho_{k}}{\phi_{k}} \leq \frac{1-\sum_{j=1}^{k-1} \rho_{j}}{\sum_{j=k}^{N} \phi_{j}}\right\} .
$$

\section{Proof}

By Lemma B.1, the set $S$ is of the form $\{1, \ldots, L\}$ for some $L$, so it suffices to show that $L=K^{*}$. First observe that

$$
\rho_{L+1}>\gamma_{L+1}=\frac{\phi_{L+1}}{\sum_{j=L+1}^{N} \phi_{j}}\left(1-\sum_{j=1}^{L} \rho_{j}\right) .
$$

By Lemma B.2 and the definition of $K^{*}$, this implies $L \geq K^{*}$.

We also have $\gamma_{L}=\rho_{L}$ and $\frac{\gamma_{L}}{\phi_{L}} \leq \frac{\gamma_{L+1}}{\phi_{L+1}}$. Thus,

$$
\rho_{L} \leq \frac{\phi_{L}}{\phi_{L+1}} \gamma_{L+1}=\frac{\phi_{L}}{\sum_{j=L+1}^{N} \phi_{j}}\left(1-\sum_{j=1}^{L} \rho_{j}\right)
$$


which is equivalent to

$$
\rho_{L} \leq \frac{\phi_{L}}{\sum_{j=L}^{N} \phi_{j}}\left(1-\sum_{j=1}^{L-1} \rho_{j}\right)
$$

By Lemma B.2 and the definition of $K^{*}$, this implies $L \leq K^{*}$.

\section{Basic GPS inequalities}

Lemma C.1 Let $S \subseteq\{1, \ldots, N\}$ be a set and let $\alpha_{j}, j \in S$, be numbers such that

$$
\alpha_{i} \frac{1-\sum_{j \in S} \phi_{j}}{\phi_{i}} \leq 1-\sum_{j \in S} \alpha_{j}
$$

for all $i \in S$.

Then

$$
\sum_{j \in S} B_{j}(r, t) \geq \sum_{j \in S} \inf _{r \leq s \leq t}\left\{A_{j}(r, s)+\alpha_{j}(t-s)\right\}
$$

for all $0 \leq r \leq t$.

\section{Proof}

For given values of $r, t$, define

$$
v^{*}:=\max _{r \leq v \leq t}\left\{v: \sum_{j \in S} B_{j}(r, v) \geq \sum_{j \in S} \inf _{r \leq s \leq v}\left\{A_{j}(r, s)+\alpha_{j}(v-s)\right\}\right\} .
$$

We need to show that $v^{*}=t$. Suppose not, i.e., $v^{*}<t$. Then there must be some source $i^{*}$ for which

$$
B_{i^{*}}(r, v)<\inf _{r \leq s \leq v}\left\{A_{i^{*}}(r, s)+\alpha_{i^{*}}(v-s)\right\}
$$

for all $v \in\left(v^{*}, w^{*}\right)$ for some $w^{*}>v^{*}$.

Define

$$
u^{*}:=\max _{r \leq u \leq w^{*}}\left\{u: B_{i^{*}}(r, u) \geq \inf _{r \leq s \leq u}\left\{A_{i^{*}}(r, s)+\alpha_{i^{*}}(u-s)\right\}\right\} .
$$

First observe that

$$
B_{i^{*}}\left(u^{*}, w^{*}\right) \leq \alpha_{i^{*}}\left(w^{*}-u^{*}\right)
$$


because otherwise

$$
\begin{aligned}
B_{i^{*}}\left(r, w^{*}\right) & =B_{i^{*}}\left(r, u^{*}\right)+B_{i^{*}}\left(u^{*}, w^{*}\right) \\
& >\inf _{r \leq s \leq u^{*}}\left\{A_{i^{*}}(r, s)+\alpha_{i^{*}}\left(u^{*}-s\right)\right\}+\alpha_{i^{*}}\left(w^{*}-u^{*}\right) \\
& =\inf _{r \leq s \leq u^{*}}\left\{A_{i^{*}}(r, s)+\alpha_{i^{*}}\left(w^{*}-s\right)\right\} \\
& \geq \inf _{r \leq s \leq w^{*}}\left\{A_{i^{*}}(r, s)+\alpha_{i^{*}}\left(w^{*}-s\right)\right\},
\end{aligned}
$$

contradicting the definition of $w^{*}$.

Further observe that

$$
B_{i^{*}}(r, u)<\inf _{r \leq s \leq u}\left\{A_{i^{*}}(r, s)+\alpha_{i^{*}}(u-s)\right\} \leq A_{i^{*}}(r, u)
$$

for all $u \in\left(u^{*}, w^{*}\right)$, so that source $i^{*}$ must be continuously backlogged during the interval $\left(u^{*}, w^{*}\right)$.

Hence, by definition of the GPS discipline,

$$
B_{i^{*}}\left(u^{*}, w^{*}\right) \geq \frac{\phi_{i^{*}}}{\phi_{j}} B_{j}\left(u^{*}, w^{*}\right)
$$

for all $j=1, \ldots, N$, and

$$
\sum_{j=1}^{N} B_{j}\left(u^{*}, w^{*}\right)=w^{*}-u^{*} .
$$

Using (13), (14),

$$
\sum_{j \notin S} B_{j}\left(u^{*}, w^{*}\right) \leq \alpha_{i^{*}} \frac{\sum_{j \notin S} \phi_{j}}{\phi_{i^{*}}}\left(w^{*}-u^{*}\right) .
$$

By virtue of (12),

$$
\alpha_{i^{*}} \frac{\sum_{j \notin S} \phi_{j}}{\phi_{i^{*}}} \leq 1-\sum_{j \in S} \alpha_{j} .
$$

From (16), (17),

$$
\sum_{j \notin S} B_{j}\left(u^{*}, w^{*}\right) \leq\left(1-\sum_{j \in S} \alpha_{j}\right)(t-s) .
$$

Combining (15), (18),

$$
\sum_{j \in S} B_{j}\left(u^{*}, w^{*}\right) \geq \sum_{j \in S} \alpha_{j}\left(w^{*}-u^{*}\right) .
$$

By definition,

$$
\sum_{j \in S} B_{j}\left(r, u^{*}\right) \geq \sum_{j \in S} \inf _{r \leq s \leq u^{*}}\left\{A_{j}(r, s)+\alpha_{j}\left(u^{*}-s\right)\right\} .
$$


From (19), (20),

$$
\begin{aligned}
\sum_{j \in S} B_{j}\left(r, w^{*}\right) & =\sum_{j \in S} B_{j}\left(r, u^{*}\right)+\sum_{j \in S} B_{j}\left(u^{*}, w^{*}\right) \\
& \geq \sum_{j \in S} \inf _{r \leq s \leq u^{*}}\left\{A_{j}(r, s)+\alpha_{j}\left(u^{*}-s\right)\right\}+\sum_{j \in S} \alpha_{j}\left(w^{*}-u^{*}\right) \\
& =\sum_{j \in S} \inf _{r \leq s \leq u^{*}}\left\{A_{j}(r, s)+\alpha_{j}\left(w^{*}-s\right)\right\} \\
& \geq \sum_{j \in S} \inf _{r \leq s \leq w^{*}}\left\{A_{j}(r, s)+\alpha_{j}\left(w^{*}-s\right)\right\}
\end{aligned}
$$

contradicting the definition of $v^{*}$, so we must have $v^{*}=t$ as required.

We now show that $\alpha_{j}=\gamma_{j E}(\delta), j \in S \supseteq S_{E}$, satisfy (12) for all $\delta \geq \delta_{0}$ for some $\delta_{0}<0$.

Lemma C.2 For any set $E \subseteq\{1, \ldots, N\}, S \supseteq S_{E}$,

$$
\gamma_{i E}(\delta) \frac{1-\sum_{j \in S} \phi_{j}}{\phi_{i}} \leq 1-\sum_{j \in S} \gamma_{j E}(\delta)
$$

for all $i \in S$ and $\delta \geq \delta_{0}$ for some $\delta_{0}<0$.

\section{Proof}

Using the definition of $\gamma_{j E}(\delta)$,

$$
\begin{aligned}
1-\sum_{j \in S} \gamma_{j E}(\delta) & =1-\sum_{j \in S_{E}} \gamma_{j E}(\delta)-\sum_{j \in S \backslash S_{E}} \gamma_{j E}(\delta) \\
& =1-\sum_{j \in S_{E}} \gamma_{j E}(\delta)-\sum_{j \in S \backslash S_{E}} \frac{\phi_{j}}{\sum_{k \notin S_{E}} \phi_{k}}\left(1-\sum_{k \in S_{E}} \gamma_{k E}(\delta)\right) \\
& =\left(1-\frac{\sum_{k \in S \backslash S_{E}} \phi_{k}}{\sum_{k \notin S_{E}} \phi_{k}}\right)\left(1-\sum_{k \in S_{E}} \gamma_{k E}(\delta)\right) \\
& =\frac{\sum_{k \notin S_{E}} \phi_{k}-\sum_{k \in S \backslash S_{E}} \phi_{k}}{\sum_{k \notin S_{E}} \phi_{k}}\left(1-\sum_{k \in S_{E}} \gamma_{k E}(\delta)\right) \\
& =\frac{1-\sum_{k \in S_{E}} \phi_{k}-\sum_{k \in S \backslash S_{E}} \phi_{k}}{\sum_{k \notin S_{E}} \phi_{k}}\left(1-\sum_{k \in S_{E}} \gamma_{k E}(\delta)\right) \\
& =\frac{1-\sum_{k \in S} \phi_{k}}{\sum_{k \notin S_{E}} \phi_{k}}\left(1-\sum_{k \in S_{E}} \gamma_{k E}(\delta)\right)
\end{aligned}
$$


Thus, we need to show that

$$
\gamma_{i E}(\delta) \leq \frac{\phi_{i}}{\sum_{j \notin S_{E}} \phi_{j}}\left(1-\sum_{j \in S_{E}} \gamma_{j E}(\delta)\right) \frac{\phi_{i}}{\sum_{j \notin S_{E}} \phi_{j}}\left(1-\sum_{j \in S_{E}}(1-\delta) \rho_{j}\right)
$$

for all $i \in S$.

By definition, the above inequality holds with equality for all $i \in S \backslash S_{E}$.

From the definition of $S_{E}$ and the equivalence relation (11),

$$
\frac{\rho_{i}}{\phi_{i}} \leq \max _{j \in S_{E}} \frac{\rho_{j}}{\phi_{j}} \leq \frac{1-\sum_{j \in S_{E}} \rho_{j}}{\sum_{j \notin S_{E}} \phi_{j}}
$$

for all $i \in S_{E}$.

Hence, for all $i \in S$ and $\delta \geq \delta_{0}$,

$$
\gamma_{i E}(\delta)=(1-\delta) \rho_{i} \leq \frac{\phi_{i}}{\sum_{j \notin S_{E}} \phi_{j}}\left(1-\sum_{j \in S_{E}}(1-\delta) \rho_{j}\right)
$$

for some $\delta_{0}<0$. 